\title{
O PAIC E A EQUIDADE NAS ESCOLAS DE ENSINO FUNDAMENTAL CEARENSES
}

\author{
PAULA KASMIRSKI \\ JOANA GUSMAO \\ VANDA RIBEIRO
}

\section{RESUMO}

O objetivo deste artigo é verificar se o Programa Alfabetização na Idade Certa (Paic) contribuiu para a melhoria da equidade nas redes municipais de ensino do estado do Ceará. Conceituou-se equidade como uma situação em que todos os alunos, independentemente de sua situação de origem, atingem níveis apropriados de resultado. Como medida de resultado, usou-se a proficiência dos alunos em Língua Portuguesa (LP) na Prova Brasil. Avaliou-se o impacto do Paic sobre a probabilidade de um aluno atingir um desempenho adequado pelo método de diferenças-em-diferenças. Concluiu-se que o Paic melhorou a equidade na coorte analisada, pois aumentou a proporção de alunos que atingem a proficiência apropriada em LP, em especial em escolas cuja maioria dos alunos é pobre. A análise da política revelou que o Paic apresenta componentes alinhados com princípios de justiça que se ajustam ao objetivo de equidade na escola de educação básica.

PALAVRAS-CHAVE EQUidAdE • AVALIAÇÃo dA EDUCAÇÃo • POLÍTICAS EDUCACIONAIS • DESIGUALDADES EDUCACIONAIS. 


\section{EL PAIC Y LA EQUIDAD EN LAS ESCUELAS DE EDUCACIÓN BÁSICA DE CEARÁ}

RESUMEN

El propósito de este artículo es verificar si el Programa de Alfabetización en la Edad Cierta (Paic) contribuyó para mejorar la equidad en las redes municipales de enseñanza del estado de Ceará. La equidad se conceptualizó como una situación en la que todos los estudiantes, independientemente de su situación de origen, alcanzan niveles adecuados de resultado. Para medir el resultado se utilizó la proficiencia de los alumnos en Lengua Portuguesa (LP) en la Prueba Brasil. Se evaluó el impacto del Paic sobre la probabilidad de que un estudiante lograra un desempeño adecuado por el método de diferencias en diferencias. La conclusión es que hubo mejora de la equidad debido al Paic en la cohorte analizada, ya que aumentó la proporción de alumnos que alcanzaron el nivel de competencia esperado en LP, sobre todo en escuelas que poseen una mayoria de estudiantes pobres. El análisis de la política reveló que el Paic presenta componentes que están en línea con principios de justicia que se ajustan al objetivo de equidad en la escuela de educación básica.

PALABRAS CLAVE EQUIDAD • EVALUACIÓN DE LA EDUCACIÓN • POLÍTICAS EDUCACIONALES • DESIGUALDADES EDUCACIONALES.

\section{PAIC AND EQUITY IN ELEMENTARY SCHOOLS IN CEARÁ}

ABSTRACT

The aim of this paper is to verify if the Programa Alfabetização na Idade Certa [Literacy in the Right Age Program] (Paic) has contributed to improving the equity in Ceará's municipal schools. Equity is defined as a situation in which all students, regardless of their social origin, achieve appropriate results. The evaluation of our results is a pedagogical interpretation that indicates adequate proficiency in the Portuguese language of students in Prova Brasil. We investigated the impact of Paic's on the probability of students achieving satisfactory performance in Portuguese using the Differences-in-Differences (DID) method. The equity concept, operationalized through DID, led us to conclude that the program improved equity in the analyzed cohort because it increased the proportion of students that reached the adequate level of Portuguese proficiency, especially in schools where the majority of students are poor. The analysis revealed that Paic presented elements aligned with principles of justice which adjust to the objective of equity in elementary school.

KEYWORDS FAIRNESS • EDUCATIONAL EVALUATION • EDUCATIONAL POLICY • EDUCATIONAL INEQUALITIES. 


\section{INTRODUÇÃO}

O estado do Ceará tem melhorado seus indicadores educacionais, como a proficiência média em Língua Portuguesa (LP) nos anos iniciais do ensino fundamental (EF1), medida pelo Sistema de Avaliação da Educação Básica (Saeb)/Prova Brasil, que tem crescido desde 2001. Até 2005, o estado acompanhou a trajetória do Nordeste, conforme indica a Figura 1.

FIGURA 1 - Proficiência média em Língua Portuguesa na 4⿳亠丷a série do ensino fundamental para as escolas urbanas municipais (SAEB 1995 a 2005)

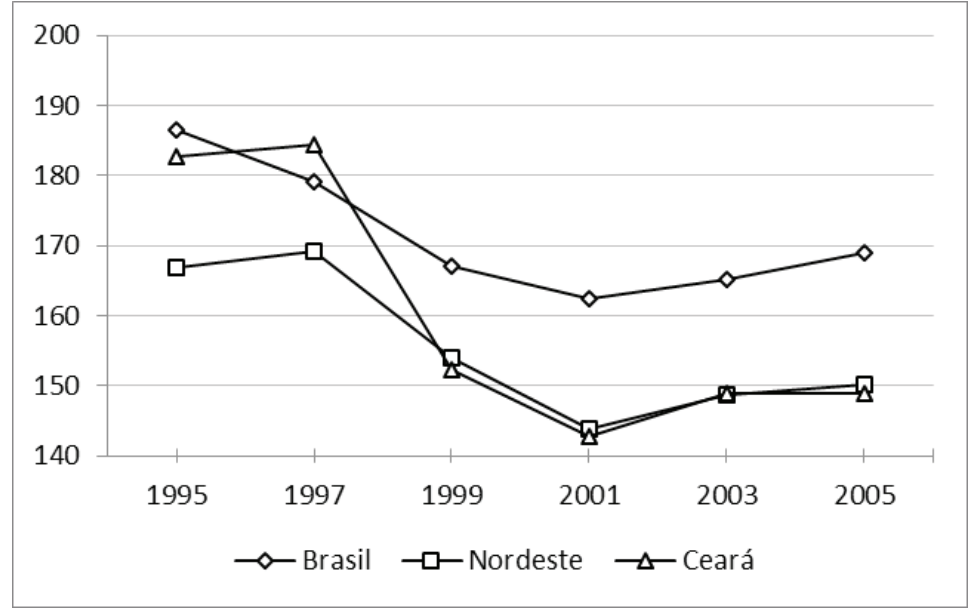

Fonte: Brasil (2007). 
Mas, a partir de 2007, a média dessa proficiência começa a descolar da regional e, em 2013, praticamente alcança o Brasil (Figura 2). Entre 2005 e 2013, a média cearense em LP cresceu $26 \%$, a nordestina, $16 \%$, e a brasileira, $13 \%$. A proficiência em Matemática e a taxa de aprovação nos anos iniciais do EF também melhoraram, impactando o Índice de Desenvolvimento da Educação Básica (Ideb) cearense da rede pública, que cresceu 78\% entre 2005 e 2013. Os Idebs do Nordeste e do Brasil cresceram, respectivamente, $52 \%$ e $36 \%$ no mesmo período.

FIGURA 2 - Proficiência média em Língua Portuguesa na 4로 série do ensino fundamental para as escolas da rede pública (Prova Brasil 2005 a 2013)

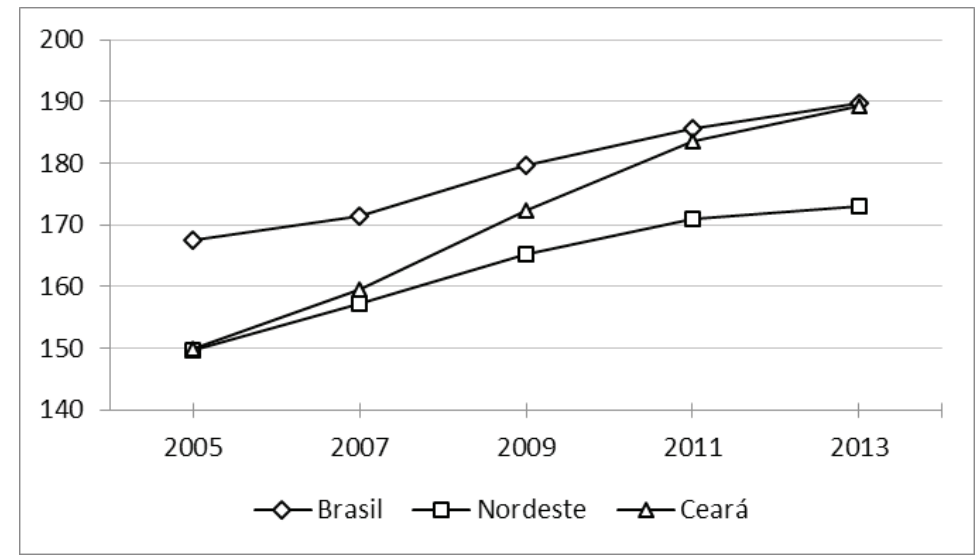

Fonte: Brasil (2015). 
TABELA 1 - Proficiência média em Língua Portuguesa e percentual de alunos que atingiu 200 pontos ou mais na Prova Brasil, por classe econômica - definida segundo o Critério Brasil, no Ceará e nos outros estados do Nordeste

\begin{tabular}{|c|c|c|c|c|c|c|c|c|c|c|c|c|}
\hline \multirow{3}{*}{$\begin{array}{l}\text { CLASSE } \\
\text { SOCIAL }\end{array}$} & \multicolumn{6}{|c|}{ PROFICIÊNCIA MÉDIA EM LP } & \multicolumn{6}{|c|}{$\begin{array}{l}\text { PORCENTAGEM DE ALUNOS COM } 200 \text { PONTOS } \\
\text { OU MAIS NA PROFICIEENCIA EM LP }\end{array}$} \\
\hline & \multicolumn{3}{|c|}{ CEARÁ } & \multicolumn{3}{|c|}{$\begin{array}{l}\text { NORDESTE } \\
\text { (SEM CEARÁ) }\end{array}$} & \multicolumn{3}{|c|}{ CEARÁ } & \multicolumn{3}{|c|}{$\begin{array}{l}\text { NORDESTE } \\
\text { (SEM CEARÁ) }\end{array}$} \\
\hline & 2007 & 2011 & $\begin{array}{c}\% \\
\text { VARIAÇÃO }\end{array}$ & 2007 & 2011 & $\begin{array}{c}\% \\
\text { VARIAÇÃO }\end{array}$ & 2007 & 2011 & $\begin{array}{c}\% \\
\text { VARIAÇÃO }\end{array}$ & 2007 & 2011 & $\begin{array}{c}\% \\
\text { VARIAÇÃO }\end{array}$ \\
\hline A ou B & 164.1 & 187.1 & 14.0 & 164.2 & 175.4 & 6.8 & 17.5 & 36.6 & 108.7 & 17.3 & 25.9 & 49.7 \\
\hline $\mathrm{C} 1$ & 165.8 & 189.2 & 14.1 & 163.5 & 174.1 & 6.5 & 18.9 & 38.7 & 104.8 & 16.3 & 24.9 & 52.8 \\
\hline $\mathrm{C} 2$ & 163.5 & 184.6 & 12.9 & 160.5 & 169.7 & 5.7 & 16.7 & 34.4 & 106.0 & 13.6 & 20.8 & 52.9 \\
\hline D & 155.2 & 176.2 & 13.5 & 154.5 & 161.5 & 4.5 & 11.1 & 26.4 & 137.2 & 9.6 & 14.4 & 50.0 \\
\hline$E$ & 144.9 & 164.6 & 13.6 & 146.3 & 153.3 & 4.8 & 7.0 & 18.3 & 161.0 & 6.5 & 9.6 & 47.7 \\
\hline Todos & 159.2 & 183.4 & 15.2 & 158.4 & 169.0 & 6.7 & 14.2 & 33.0 & 133.0 & 12.7 & 20.5 & 61.4 \\
\hline
\end{tabular}

Fonte: Elaboração das autoras com dados da Prova Brasil/Inep.

A Tabela 1 mostra que o aumento da proficiência média em LP no Ceará ocorreu em todas as classes econômicas de modo proporcional (em torno de $13 \%$ ) e que o estado mais que dobrou o percentual de alunos que atinge 200 pontos ou mais na escala do Saeb, tanto em termos gerais, quanto por classes, sendo que as crianças de menor nível socioeconômico (NSE) apresentaram maior crescimento. O nível de 200 pontos é considerado um indicador de patamar adequado de conhecimento em LP (TODOS PELA EDUCAÇÃO - TPE, 2007). A região Nordeste, sem o Ceará, também melhorou, porém bem menos que o Brasil. Vale destacar que o percentual de estudantes com proficiência apropriada é muito baixo, tanto no Nordeste quanto no Ceará, demonstrando o desafio que ainda existe em torno da equidade.

Há razões para crer que a melhoria dos indicadores educacionais cearenses, em especial na proficiência de LP, foi causada, pelo menos parcialmente, pela política pública. A evolução de indicadores socioeconômicos do Ceará foi semelhante ao padrão observado na região Nordeste (PADILHA et al., 2013) e, nas últimas duas décadas, o governo do estado do Ceará vem 
implementando uma política do tipo "descentralização orquestrada” do EF1 (PADILHA; BATISTA, 2013). Nesse modelo, os municípios assumem a responsabilidade pelo ensino em colaboração com o estado, que apoia a implementação das políticas, sobretudo por meio das Coordenadorias Regionais de Desenvolvimento da Educação (Credes). Em 2007, foi adotado, como política pública, o Programa de Alfabetização na Idade Certa (Paic), cujo foco é alfabetizar todos os alunos de até 7 anos de idade.

O objetivo deste artigo é verificar se o Paic contribuiu para a melhoria da equidade em termos de proficiência em LP no EF1 nas redes municipais cearenses. Nosso interesse recai sobre o Paic porque essa foi a política na qual o governo se centrou no período estudado por Padilha et al. (2013) e em que os resultados melhoraram e destoaram do padrão nordestino.

Padilha et al. (2013) usaram modelagem multinível e constataram o ganho de equidade no EF1 na rede pública desse estado, com redução do peso do NSE dos alunos sobre o Ideb das escolas, entre 2007 e 2011, controlando o NSE dos municípios. ${ }^{1}$ Esse trabalho e outros (PADILHA; BATISTA, 2013; PADILHA et al., 2011, 2012) apontaram para a necessidade de compreender melhor as políticas do Ceará e verificar se elas ajudam a explicar tanto a melhoria da qualidade quanto da equidade nas redes de ensino do estado.

O Paic foi avaliado por Costa e Carnoy (2014) e Lavor e Arraes (2014). Ambos os trabalhos estimaram o efeito de tratamento do programa sobre a proficiência média dos alunos, usando métodos que controlam por características não observáveis - diferenças triplas e controle sintético, respectivamente - e encontraram efeito positivo e significativo sobre o desempenho em LP.

Antes de apresentarmos o conceito de equidade usado neste artigo, precisamos discutir brevemente a perspectiva adotada, apoiada em Ribeiro (2012). Com base em Crahay (2000) e Dubet (2009), Ribeiro (2014) explica que o conceito de equidade fundamenta-se na ideia central de que a meritocracia é um princípio de justiça incompatível com a educação básica. Isso se deve a dois fatos: (i) a educação básica é
1 Esses autores usaram a classificação feita pelo Instituto Brasileiro de Geografia e Estatística (IBGE) das cidades que provê uma hierarquia dos centros urbanos, de acordo com seu grau de influência socioeconômica (PADILHA et al., 2013). 
direito obrigatório; (ii) há correlação entre desigualdade social e desigualdade escolar. Logo, as crianças sozinhas não têm governabilidade por seus rendimentos, de forma independente de suas situações de origem, fazendo com que o talento não seja bom preditor da distribuição de conhecimento. Crahay (2000) e Dubet (2009) apregoam um novo princípio de justiça para a escolarização básica: a igualdade de base ou igualdade de conhecimentos adquiridos. Segundo tais princípios, todas as crianças na educação básica devem ter acesso ao que a sociedade definiu como necessário ser aprendido, de modo que tenham garantidas sua dignidade, autoestima e trajetória escolar menos turbulenta, diminuindo, assim, a correlação entre desigualdade escolar e social. Conforme afirma Ribeiro (2012), esse princípio de justiça pode ser fundamentado nas ideias de Rawls (2003), para quem o princípio de justiça é considerado equidade, sem foco no talento, como única base de legitimidade nas distribuições de bens, que melhor garante a cooperação social no tipo de sociedade em que vivemos, propícia à produção contínua de desigualdades, mas também aberta a admitir, como legítimos, os conflitos de interesses e as lutas por direitos que diminuem essas desigualdades.

O conceito de equidade usado no texto é o operacionalizado por Ribeiro (2012), com base nos autores acima mencionados: uma situação em que todos os alunos, independentemente de sua situação de origem, atingem níveis adequados de resultados. Isso não significa que todos devem apresentar o mesmo resultado, mas sim que, idealmente, sua distribuição deve ser truncada à esquerda no valor definido como adequado. Todas as crianças atingiriam no mínimo esse patamar de resultado e, para além dele, pode haver diferenças. Assim, é possível dividir a análise da equidade em duas partes: na primeira, pode-se averiguar que fatores explicam a probabilidade de um aluno atingir um nível adequado de resultado; na segunda parte, pode-se averiguar o quão desigual é a distribuição do resultado para aqueles que atingiram esse patamar.

Neste artigo, vamos debruçar-nos sobre a primeira parte, usando como resultado a proficiência em LP e como nível adequado o valor de 200 pontos na escala do Saeb, definido pelo 
Todos Pela Educação (TPE), com base no desempenho médio dos países da Organização para a Cooperação e Desenvolvimento Econômico (OCDE) em 2006 (TPE, 2007). Pode-se pensar nesse valor como um piso para a nota apropriada, visto que é complexa a tarefa de fixar tal valor. Vale mencionar que uma avaliação mais completa da equidade na escola deve levar em conta outros resultados que sejam relevantes, porém a proficiência em leitura é central para atingir outros fins educacionais.

\section{O PAIC}

O Paic é definido pela Secretaria Estadual de Educação do Ceará (Seduc-CE) (CEARÁ, 2012) como uma política de cooperação entre estado e municípios. Originalmente, seu objetivo geral era alfabetizar todos os alunos das redes públicas de ensino do Ceará de até 7 anos de idade. ${ }^{2} \mathrm{O}$ foco na alfabetização está atrelado à história do programa, que tem origem em 2004, com o Comitê Cearense para Eliminação do Analfabetismo Escolar (CCEA). Presidido pela Assembleia Legislativa e com a participação de universidades e instituições da sociedade civil, o CCEA realizou uma pesquisa para avaliar o nível de alfabetização de alunos da segunda série do EF e constatou que apenas $15 \%$ das crianças estavam alfabetizadas. Pode-se dizer que o resultado dessa avaliação mobilizou atores diversos e incentivou a articulação de uma rede de parceiros para buscar e experimentar soluções (GUSMÃO; RIBEIRO, 2011).

Essa articulação gerou o Paic, cujo objetivo era apoiar os municípios cearenses no combate ao analfabetismo escolar no EF1. A primeira fase do programa, coordenada pela Associação dos Municípios e Prefeitos do Estado do Ceará (Aprece) e União dos Dirigentes Municipais de Educação do Ceará (Undime-CE), com parceria do Fundo das Nações Unidas para a Infância (Unicef), atingiu 56 municípios e aconteceu em 2005 e 2006. Nessa etapa, a principal ação desenvolvida foi a avaliação diagnóstica dos níveis de leitura e escrita dos alunos da $2^{\mathrm{a}}$ série (GUSMÃO; RIBEIRO, 2011).

Em 2007, a Seduc assumiu a coordenação do Paic. O desenho da política implantada a partir de então até o final da
2 ○ programa foi ampliado em 2011 (Paic+), passando a abranger ações para o ensino de Matemática e demais anos do EF1 (CEARÁ, 2015b). 
primeira gestão de Cid Gomes (2007 a 2010) baseou-se na experiência acumulada pelo Comitê e pela primeira fase do programa. A experiência do município de Sobral, que implantou política parecida já no começo dos anos 2000, também influenciou o Paic, pois seus gestores estavam na Seduc em 2007 (GUSMÃO; RIBEIRO, 2011).

O programa implantando a partir de 2007 prevê ações para o $1^{\circ}$ e o $2^{\circ}$ ano do EF de 9 anos e está organizado em cinco eixos: gestão da educação municipal, avaliação externa, alfabetização, formação do leitor e educação infantil, cujas ações ocorrem

[...] por meio de encontros de formação, acompanhamento técnico, tanto a distância quanto in loco, e disponibilização de instrumentos e materiais. Municípios considerados prioritários têm apoio reforçado. (SEDUC-CE, 2012, p. 21)

Todos os municípios do estado aderiram à política. Importante mencionar que o EF1, em 2007, já estava quase que universalmente municipalizado no estado.

As primeiras ações de todos os eixos começaram efetivamente em 2008, conforme informado pela Seduc no site do Paic. No eixo da alfabetização, por exemplo, houve, em 2008: formação de professores alfabetizadores multiplicadores para turmas de $1^{\circ}$ ano; formação direta de docentes alfabetizadores do $2^{\circ}$ ano; produção e impressão de material estruturado para alfabetização de alunos do $1^{\circ}$ ano; e aquisição de material estruturado para alunos do $2^{\circ}$ ano (CEARÁ, 2015a).

3 "Para levantar informações sobre - Paic, [as autoras] leram materiais variados e realizaram entrevistas semiestruturadas com diferentes atores engajados no programa. [...] As entrevistas dos atores municipais foram realizadas nos municípios de Fortim e Canindé [...]. Uma primeira versão da publicação foi discutida e aprimorada em oficina de trabalho com um grupo técnico, composto por membros da Seduc, representantes dos municípios e parceiros do Paic Em seguida, uma nova versão fo submetida a um grupo consultivo de especialistas, o que permitiu seu aperfeiçoamento" (SEDUC-CE, 2012

p. 34-35)

\section{POR QUE O PAIC PODE TER AUMENTADO A EQUIDADE}

O objetivo desta seção é descrever com mais detalhes algumas ações específicas do Paic que podem ter afetado a equidade. Ribeiro $(2012,2014)$ discute princípios de justiça para a educação básica que se ajustam ao objetivo de equidade na escola e aponta práticas e elementos que constituem políticas capazes de gerar equidade. Porém, gostaríamos de explicitar que nossa principal fonte de informações sobre o Paic (SEDUC-CE, 2012) é fruto da sistematização ${ }^{3}$ e que, acreditamos, 
é capaz de captar aspectos do desenho da política e de sua implementação. Conforme aponta Holliday (2012, p. 17),

[...] a sistematização de experiências é uma interpretação crítica de uma ou várias experiências que, a partir da sua ordenação e reconstrução, descobre ou explicita a lógica do processo vivido: os factores que intervieram, como se relacionam entre si e por que é que sucederam dessa forma.

Para Crahay (2000), a promoção de uma escola mais equitativa exige o estabelecimento de quais são as competências que os alunos precisam adquirir. Essa ideia baseia-se em pesquisas que mostram que quando os professores não organizam o ensino em torno de uma dada competência, os alunos apresentam dificuldades em sua aquisição. Ensino sem objetivo de aprendizagem não seria ensino propriamente dito. Embora fundamental, o estabelecimento das competências não é, entretanto, suficiente. Seria necessária, também, a regulação da ação dos professores por meio do dispositivo que nominou "sistema de pilotagem", focado no acompanhamento da aprendizagem das competências essenciais predefinidas. Trata-se de um sistema que prevê a avaliação externa e que gera interpretação relativa ao que cada aluno aprendeu, onde estão as dificuldades de cada um em relação ao previsto. Esse saber deve chegar até o professor de modo que possa adotar medidas para solucionar os problemas e corrigir os rumos. Crahay (2000) afirma que "conselheiros" podem apoiar os professores na tarefa de analisar os resultados dessas avaliações e de implantar os ajustes pedagógicos necessários.

De acordo com Ribeiro (2014), que se baseou em Crahay (2000) e Dubet (2009), para o alcance de maiores níveis de equidade, deve haver vigilância tanto dos processos de implementação da política que geram a distribuição do bem social educação escolar, como também dos resultados, ou seja, daquilo que foi distribuído. A autora afirma que, quando a política é implementada, há conflitos de interesse, razão pela qual o desenho de uma política não garante sua implementação tal como foi inicialmente prevista. Assim, se seu desenho objetiva distribuição entre todos, a "vigilância" 
sobre os processos com vistas a remanejamentos é fundamental. Mas zelar somente pelos processos não seria suficiente, pois é a distribuição realizada que indica se uma política contribuiu ou não para a equidade. Segundo Ribeiro (2014), os processos precisam ser cotejados frente a seus resultados para que se efetuem correções de rumo. Ainda na aferição dessa distribuição, a avaliação em larga escala assume papel de relevância desde que usada não somente para a produção de médias, mas para acompanhar o desempenho de todos, uma vez que denota parte do que se define ser necessário aprender quando se cursa a educação básica.

As informações apresentadas a seguir indicam que o conjunto de ações do Paic se aproxima do que Crahay (2000) chama de "sistema de pilotagem". Há também indícios de vigilância sobre processos e resultados, conforme as ponderações de Ribeiro (2012, 2014).

Algumas das evidências de que o Paic congrega ações de vigilância, tanto dos processos de implementação quanto dos seus resultados, são as ações de planejamento e acompanhamento nas escolas, secretarias municipais, Credes e Coordenadoria de Cooperação com os Municípios (Copem) (área da Seduc responsável pelo programa). Trata-se de uma cadeia de vigilância com apoio para reestruturação de ações que parte da Seduc, intermediada pelas Credes e secretarias municipais, que chega às escolas e salas de aula. Na Seduc, instituiu-se a Copem, que conta com técnicos que se dividem entre os cinco eixos do programa. Esses técnicos realizam formação para os técnicos das Credes que, por sua vez, fazem formação para as redes municipais. A perspectiva da vigilância sobre processos pode ser verificada também por meio da ação da Seduc de complementar o salário de um técnico das secretarias municipais, que se torna responsável por apoiar a estruturação desses órgãos e também por apoiar as escolas na implementação das linhas de ação do Paic. Ademais, técnicos da Seduc também visitam diretamente aqueles municípios que apresentam mais dificuldade em cumprir suas metas. Nessas visitas, prefeitos e secretários são envolvidos, mostram-se os dados do município e buscam-se acordos com vistas à correção dos problemas. 
No que tange à vigilância dos resultados, outro preditor da possibilidade da produção da equidade, segundo Ribeiro (2012), o Paic desenvolveu um sistema de monitoramento da aprendizagem composto pela Prova Paic (de caráter diagnóstico, avalia leitura e escrita e é aplicada no início do ano), pelo Sistema Permanente de Avaliação da Educação Básica do Estado do Ceará (Spaece-Alfa) (avaliação externa estadual que ocorre ao final do ano letivo e que avalia leitura) e por instrumentos usados pelas escolas, equipes municipais e estaduais ao longo de todo o ano letivo. Há instrumentos que mapeiam a situação de leitura, a frequência e a quantidade de títulos de literatura infantil lidos pelas crianças do $1^{\circ} \mathrm{e}$ do $2^{\circ}$ ano bimestralmente; que acompanham a frequência de professores e demais funcionários das escolas, a utilização dos materiais estruturados pelos professores do $1^{\circ}$ e do $2^{\circ}$ ano e a participação desses nas formações; e, ainda, que verificam o aproveitamento dos dias e horas letivas (SEDUC-CE, 2012). As provas são aplicadas aos alunos e há posterior interpretação pedagógica para verificação de problemas, visando à correção de estratégias de ensino. Uma série de processos foi desenhada para acompanhamento das ações do programa com base nos resultados das avaliações externas, tendo sempre como referência as metas dos municípios. Segundo a Seduc-CE (2012, p. 10), "os processos ocorrem nos seguintes níveis: escola - escola; município - escolas; Crede - municípios; e Seduc - Credes/municípios”.

Em 2004, pesquisa do CCEA, que pode ser tomada como prenúncio do estabelecimento de um sistema de pilotagem da educação no estado, mostrou que os professores tinham pouco conhecimento teórico e metodológico sobre alfabetização infantil, por conta de falhas na formação inicial. Foi adotada, então, a estratégica de distribuição de material estruturado, objetivando

[...] retomar o uso de metodologias de alfabetização de crianças em sala de aula, tendo como referência materiais que oferecessem uma estrutura metodológica para o professor desenvolver seu trabalho. (SEDUC-CE, 2012, p. 123) 
Esses materiais pautam formações docentes e permitem abordar a alfabetização de forma mais concreta.

O material estruturado pode ajudar também o aluno, na medida em que articula o tempo com as atividades indicadas, com a marcação mais clara da rotina pedagógica e comum à pedagogia mais explícita. Assim, contribuiu-se para que o professor tenha mais clareza sobre o que está ensinando e para que o aluno saiba o que está aprendendo (SEDUC-CE, 2012).

Segundo Bernstein (1984), as crianças de NSE mais baixo tendem a aprender mais quando se adota uma pedagogia mais visível - que explicita o processo de ensino e os critérios de avaliação usados pelo professor. A não explicitação desses elementos, cuja compreensão seria central para a aprendizagem, favoreceria apenas as crianças cujas famílias já dominam os códigos de funcionamento da instituição escolar. À luz desse autor, pode-se considerar que a adoção de material estruturado, nesse contexto, também seria uma prática favorável à equidade.

O desenho do Paic prevê, ainda, incentivo à ampliação da educação infantil, com elaboração de propostas pedagógicas e formação de professores. Pesquisas como a de Campos (1997) apontam que frequentar a educação infantil aumenta o desempenho dos alunos nos EF1, sobretudo dos que têm menor NSE.

Segundo Ribeiro $(2012,2014)$, no tipo de sociedade em que vivemos, que produz desigualdade o tempo todo, quando a política é implementada, uma série de conflitos e problemas se mostram, fazendo com que objetivos almejados possam não se concretizar. O Paic tem, conforme mencionado, estratégias de acompanhamento para correções de "rotas". O bom funcionamento dessas estratégias teria, assim, possibilidade de ampliar o alcance de seus objetivos: garantir a alfabetização de todas as crianças no que se considera ser a idade certa. Tais estratégias poderiam, também, favorecer o alcance das demais habilidades consideradas adequadas para o final dos EF1, conforme a mensuração da Prova Brasil. 


\section{AVALIAÇÃO DO EFEITO DO PAIC}

Usamos dados dos Censos Escolares 2008 e 2011 para mapear a população tratada e da Prova Brasil 2007 e 2011 para estimar o efeito da política. Vamos avaliar o efeito da $2^{\mathrm{a}}$ fase do Paic, que teve início efetivo em 2008, conforme apontado na seção "O Paic”. De acordo com os Censos Escolares (Brasil, 2015), dos mais de $141 \mathrm{mil}$ alunos do $2^{\circ}$ ano do EF em 2008 nas redes municipais, somente 85.585 podem ter feito a Prova Brasil 2011 no Ceará, pois uma parcela foi reprovada em pelo menos um ano (27,73\%) e não chegou ao $5^{\circ}$ ano em 2011 e outra migrou do estado ou saiu da rede pública $(11,70 \%)$. Outra limitação dos dados diz respeito ao fato de que, em 2007, a Prova Brasil só cobria áreas urbanas, ${ }^{4}$ fato que reduz o número de alunos tratados para 28.428. Assim, o efeito do Paic sobre a coorte mencionada está condicionado por esses fatos.

Para se avaliar o efeito do programa sobre a variável de resultado, precisa-se de um grupo de controle adequado. Como, em 2007, o Paic foi universalizado - e todas as crianças das redes municipais ${ }^{5}$ foram beneficiárias da política -, 0 grupo de controle terá de ser selecionado em outros lugares com perfil semelhante ao do Ceará - demais estados nordestinos. O Ceará é um estado típico do Nordeste em termos de renda mensal média e percentual de pobres, por exemplo, e a região Nordeste destoa do resto do país. Desse modo, somente estados nordestinos parecem ser adequados para pertencer ao grupo de controle. Buscamos verificar também se os estados mais próximos ao Ceará são mais parecidos com ele. Construímos dois tipos de grupo de controle, tendo em conta o que foi discutido neste parágrafo e no anterior (Quadro 1).

As características médias dos grupos podem ser vistas na Tabela 2, na qual se pode constatar a semelhança entre os alunos das redes municipais do Ceará e os dos demais estados do Nordeste e estados vizinhos, tanto entre os alunos de interesse (não repetentes e que sempre estudaram em rede pública) quanto entre todos (geral) no período pré-tratamento (2007). Também é possível notar que os alunos de interesse têm proficiência e perfil socioeconômico (percentuais com atraso escolar, que trabalha fora de casa e que frequentou educação infantil) ligeiramente maiores que o total.
4 Apesar de constar do site do Instituto Nacional de Estudos e Pesquisas Educacionais Anísio Teixeira (Inep) que em 2007 passaram a participar da Prova Brasil escolas públicas rurais que ofertam os anos iniciais do EF, nos microdados, apenas $2 \%$ dos alunos cearenses eram de escolas da zona rural.

5 A rede estadual também foi tratada, porém, como o percentual de matrículas da coorte de interesse dessa rede no Ceará é muito pequeno desconsideramos tais matrículas na análise. 
QUADRO 1 - Descrição do grupo tratamento e dos grupos controle

\begin{tabular}{|c|l|}
\hline \multicolumn{1}{|c|}{ GRUPO } & \multicolumn{1}{c|}{ DESCRIÇÃo } \\
\hline Tratamento & $\begin{array}{l}\text { Alunos do 5ªno do EF do Ceará de redes municipais que } \\
\text { indicaram, no questionário contextual, não ter reprovações } \\
\text { e ter estudado sempre em rede pública. }\end{array}$ \\
\hline Controle 1 & $\begin{array}{l}\text { Alunos do 5ªno do EF de redes municipais de estados } \\
\text { nordestinos (Maranhão, Piaú, Pernambuco, Paraíba, Rio } \\
\text { Grande do Norte, Alagoas, Sergipe e Bahia) que indicaram, } \\
\text { no questionário contextual, não ter reprovações e ter } \\
\text { estudado sempre em rede pública. }\end{array}$ \\
\hline Controle 2 & $\begin{array}{l}\text { Alunos do 5ªno do EF de redes municipais de estados } \\
\text { vizinhos ao Ceará (Piauí, Pernambuco, Paraíba e Rio Grande } \\
\text { do Norte) que indicaram, no questionário contextual, não } \\
\text { ter reprovações e ter estudado sempre em rede pública. }\end{array}$ \\
\hline
\end{tabular}

Fonte: Elaboração das autoras.

Notas: Só foram usados dados das escolas que participaram das avaliações nos dois anos.

Com base na discussão da Introdução, o conceito de equidade foi operacionalizado do seguinte modo: estimamos o efeito do Paic sobre a probabilidade de um aluno atingir 200 pontos na escala de proficiência do Saeb em LP, piso definido pelo TPE. Também verificamos se o efeito do Paic foi diferente em escolas com maior proporção de alunos pobres, ou seja, buscamos averiguar se o programa afeta diferentemente alunos de distintas classes sociais.

Da Tabela 3 constam os percentuais de alunos que atingiram o piso referido por características sociais. Chamam atenção o fato de os alunos cearenses terem quase sempre melhorado mais que os grupos de controle e o fato de os grupos sociais com menor percentual de desempenho adequado em 2007 crescerem mais no período. Esses são os alunos das classes D e E, meninos, não negros, atrasados, que trabalham fora e que não cursaram a educação infantil.

Aplicamos o método de diferenças-em-diferenças que

$6 \bigcirc$ efeito de tratamento sobre os tratados é a média dos efeitos de cada indivíduo que faz parte da subpopulação que participou da política, ou seja, $E\left[\alpha_{i} \mid d_{i}=1\right]$, onde $\alpha_{i}$ é o efeito de tratamento no indivíduo $i$ e $d_{i}$ é uma dummy de tratamento (BLUNDELL; DIAS, 2009) identifica, geralmente, o efeito de tratamento sobre os tratados $^{6}$ e tem duas hipóteses de identificação principais: os grupos de tratamento e controle apresentam tendência temporal igual na ausência da política e os grupos não têm grandes diferenças em sua composição (BLUNDELL; DIAS, 2009). A seleção dos grupos de controle entre os estados nordestinos e a restrição da análise à coorte de alunos que não repetiram e não saíram da rede pública buscam assegurar a semelhança entre os grupos de tratados e não tratados. Quanto à hipótese de tendências temporais iguais, em princípio, poderíamos 
ter realizado um teste de tendências paralelas para avaliar sua razoabilidade, mas o teste não é factível.7 Também não foi verificado se há programas ou políticas semelhantes ao Paic nas redes municipais do grupo de controle. Tal levantamento mostrou-se inviável, tendo em vista que existem mais de 1.600 municípios no grupo. A existência de tais políticas

7 Para realizar o teste, precisariamos de dados para anos anteriores, como 2005 ou 2003, por exemplo. Contudo os dados da Prova Brasil 2005 não estão disponíveis no site do Inep e dados anteriores não são compatíveis (o Saeb é amostral, enquanto a Prova Brasil é censitária). poderia enfraquecer a hipótese de tendências paralelas e os resultados de nossa análise. 
TABELA 2 - Medidas referentes aos alunos do $5^{\circ}$ ano do ensino fundamental para os grupos de tratamento e controle, considerando-se o grupo de alunos não repetentes e que sempre estudaram na rede pública (coorte) e o grupo total de alunos (geral)

\begin{tabular}{|c|c|c|c|c|c|c|c|}
\hline \multirow{2}{*}{ VARIÁVEL } & \multirow{2}{*}{ GRUPO } & \multicolumn{3}{|c|}{ COORTE } & \multicolumn{3}{|c|}{ GERAL } \\
\hline & & 2007 & 2011 & \% VARIAÇÃO & 2007 & 2011 & \% VARIAÇÃO \\
\hline \multirow{3}{*}{ Número de alunos } & Tratamento & 34.859 & 34.119 & - & 74.043 & 75.029 & - \\
\hline & Controle 1 & 119.079 & 103.339 & - & 292.629 & 298.098 & - \\
\hline & Controle 2 & 47.181 & 41.352 & - & 113.337 & 114.981 & - \\
\hline \multirow{3}{*}{$\begin{array}{l}\% \text { de alunos que atingiu } \\
200 \text { pontos ou mais em LP }\end{array}$} & Tratamento & 18.4 & 43.3 & 135.7 & 14.2 & 33.0 & 133.0 \\
\hline & Controle 1 & 17.0 & 29.1 & 71.1 & 12.7 & 20.5 & 60.9 \\
\hline & Controle 2 & 16.9 & 30.6 & 81.7 & 12.4 & 21.6 & 73.7 \\
\hline \multirow{3}{*}{ Proficiência média em LP } & Tratamento & 165.6 & 194.5 & 17.4 & 159.2 & 183.4 & 15.2 \\
\hline & Controle 1 & 164.9 & 179.5 & 8.9 & 158.4 & 169.0 & 6.7 \\
\hline & Controle 2 & 164.2 & 181.2 & 10.4 & 157.4 & 170.1 & 8.1 \\
\hline \multirow{3}{*}{ \% da Classe A ou B } & Tratamento & 8.5 & 10.5 & 24.4 & 8.9 & 12.6 & 42.7 \\
\hline & Controle 1 & 10.6 & 13.1 & 23.0 & 10.5 & 14.5 & 37.8 \\
\hline & Controle 2 & 10.5 & 12.8 & 22.4 & 10.4 & 14.5 & 39.7 \\
\hline \multirow{3}{*}{ \% da Classe $\mathrm{C} 1$} & Tratamento & 17.5 & 23.5 & 34.3 & 16.4 & 23.0 & 40.2 \\
\hline & Controle 1 & 20.6 & 25.4 & 23.3 & 19.1 & 24.2 & 26.7 \\
\hline & Controle 2 & 21.1 & 26.7 & 26.5 & 19.8 & 25.7 & 29.8 \\
\hline \multirow{3}{*}{ \% da Classe C2 } & Tratamento & 33.0 & 41.0 & 24.2 & 30.4 & 38.0 & 25.0 \\
\hline & Controle 1 & 34.0 & 36.6 & 7.6 & 32.0 & 34.0 & 6.3 \\
\hline & Controle 2 & 35.7 & 38.7 & 8.4 & 33.7 & 36.0 & 6.8 \\
\hline \multirow{3}{*}{ \% da Classe D } & Tratamento & 33.4 & 22.3 & -33.3 & 33.8 & 22.1 & -34.6 \\
\hline & Controle 1 & 29.0 & 21.6 & -25.3 & 30.1 & 21.9 & -27.3 \\
\hline & Controle 2 & 28.0 & 19.2 & -31.3 & 29.6 & 19.6 & -33.6 \\
\hline \multirow{3}{*}{ \% da Classe E } & Tratamento & 7.6 & 2.5 & -66.6 & 10.0 & 3.1 & -69.5 \\
\hline & Controle 1 & 5.8 & 3.1 & -46.4 & 7.9 & 4.2 & -47.3 \\
\hline & Controle 2 & 4.7 & 2.3 & -51.0 & 6.3 & 3.0 & -51.9 \\
\hline \multirow{3}{*}{$\%$ de meninas } & Tratamento & 51.1 & 51.1 & 0.0 & 45.2 & 45.4 & 0.4 \\
\hline & Controle 1 & 52.7 & 52.1 & -1.1 & 46.3 & 45.2 & -2.5 \\
\hline & Controle 2 & 52.7 & 52.4 & -0.6 & 46.9 & 45.5 & -2.9 \\
\hline \multirow{3}{*}{$\%$ de pretos ou pardos } & Tratamento & 62.7 & 61.6 & -1.8 & 60.3 & 59.2 & -1.9 \\
\hline & Controle 1 & 62.4 & 61.0 & -2.2 & 61.1 & 58.6 & -4.1 \\
\hline & Controle 2 & 58.7 & 58.2 & -0.8 & 57.6 & 56.0 & -2.7 \\
\hline \multirow{3}{*}{$\%$ com atraso escolar } & Tratamento & 18.8 & 11.2 & -40.8 & 31.2 & 28.3 & -9.3 \\
\hline & Controle 1 & 24.1 & 16.2 & -32.8 & 38.3 & 32.7 & -14.7 \\
\hline & Controle 2 & 22.9 & 14.4 & -37.2 & 37.0 & 31.5 & -15.0 \\
\hline \multirow{3}{*}{$\%$ que trabalha fora de casa } & Tratamento & 13.9 & 10.5 & -24.5 & 16.5 & 14.2 & -14.2 \\
\hline & Controle 1 & 14.5 & 12.6 & -13.3 & 17.6 & 17.3 & -1.7 \\
\hline & Controle 2 & 14.1 & 12.6 & -10.4 & 17.3 & 17.4 & 0.5 \\
\hline \multirow{3}{*}{$\%$ que frequentou El } & Tratamento & 69.4 & 82.3 & 18.6 & 64.5 & 77.5 & 20.2 \\
\hline & Controle 1 & 68.4 & 76.9 & 12.5 & 63.4 & 70.9 & 11.8 \\
\hline & Controle 2 & 70.2 & 78.4 & 11.8 & 65.7 & 72.6 & 10.4 \\
\hline
\end{tabular}

Fonte: Elaboração das autoras com dados da Prova Brasil/Inep. 
TABELA 3 - Percentuais de alunos que atingiram 200 pontos ou mais de proficiência dos grupos de tratamento e controles (coorte) e geral por características sociais - nível dos alunos

\begin{tabular}{|c|c|c|c|c|c|c|c|}
\hline \multirow[t]{2}{*}{ VARIÁVEL } & \multirow[b]{2}{*}{ GRUPO } & \multicolumn{3}{|c|}{ COORTE } & \multicolumn{3}{|c|}{ GERAL } \\
\hline & & 2007 & 2011 & $\begin{array}{c}\% \\
\text { VAR. }\end{array}$ & 2007 & 2011 & $\begin{array}{c}\% \\
\text { VAR. }\end{array}$ \\
\hline \multirow{3}{*}{ Classe A ou B } & Tratamento & 21.2 & 44.4 & 109.3 & 17.5 & 36.6 & 108.7 \\
\hline & Controle 1 & 21.4 & 33.8 & 57.7 & 17.3 & 25.9 & 49.9 \\
\hline & Controle 2 & 20.3 & 35.2 & 73.6 & 16.4 & 27.0 & 64.5 \\
\hline \multirow{3}{*}{ Classe C1 } & Tratamento & 23.3 & 48.3 & 107.3 & 18.9 & 38.7 & 104.8 \\
\hline & Controle 1 & 21.0 & 33.4 & 59.0 & 16.3 & 25.0 & 53.4 \\
\hline & Controle 2 & 20.5 & 35.0 & 70.7 & 15.7 & 25.7 & 63.7 \\
\hline \multirow{3}{*}{ Classe C2 } & Tratamento & 20.6 & 44.9 & 118.0 & 16.7 & 34.5 & 106.6 \\
\hline & Controle 1 & 17.9 & 29.5 & 64.8 & 13.6 & 20.8 & 52.9 \\
\hline & Controle 2 & 17.6 & 30.5 & 73.3 & 13.4 & 21.5 & 60.4 \\
\hline \multirow{3}{*}{ Classe D } & Tratamento & 15.0 & 36.6 & 144.7 & 11.1 & 26.4 & 137.2 \\
\hline & Controle 1 & 13.3 & 22.3 & 68.3 & 9.6 & 14.4 & 49.8 \\
\hline & Controle 2 & 13.2 & 23.4 & 77.0 & 9.2 & 15.3 & 67.2 \\
\hline \multirow{3}{*}{ Classe E } & Tratamento & 9.8 & 28.0 & 184.9 & 7.0 & 18.3 & 161.0 \\
\hline & Controle 1 & 8.6 & 17.1 & 97.6 & 6.5 & 9.6 & 47.0 \\
\hline & Controle 2 & 9.0 & 18.9 & 109.4 & 6.3 & 10.5 & 66.3 \\
\hline \multirow{3}{*}{ Menina } & Tratamento & 21.4 & 48.7 & 127.4 & 17.7 & 39.8 & 125.0 \\
\hline & Controle 1 & 19.9 & 33.9 & 70.8 & 15.9 & 26.3 & 65.4 \\
\hline & Controle 2 & 19.7 & 35.6 & 80.6 & 15.5 & 27.7 & 78.5 \\
\hline \multirow{3}{*}{ Menino } & Tratamento & 16.2 & 38.7 & 139.7 & 12.2 & 29.0 & 137.6 \\
\hline & Controle 1 & 14.7 & 25.0 & 70.3 & 10.7 & 17.0 & 59.3 \\
\hline & Controle 2 & 14.7 & 26.5 & 80.8 & 10.5 & 18.0 & 71.3 \\
\hline \multirow{3}{*}{ Negro } & Tratamento & 19.6 & 44.4 & 126.4 & 15.3 & 34.7 & 127.2 \\
\hline & Controle 1 & 17.9 & 30.1 & 68.1 & 13.6 & 21.7 & 60.0 \\
\hline & Controle 2 & 17.5 & 31.5 & 80.4 & 12.9 & 22.6 & 74.6 \\
\hline \multirow{3}{*}{ Não negro } & Tratamento & 17.5 & 42.6 & 142.7 & 14.0 & 32.7 & 134.0 \\
\hline & Controle 1 & 16.8 & 28.7 & 70.6 & 12.6 & 20.6 & 63.7 \\
\hline & Controle 2 & 17.3 & 30.5 & 76.5 & 13.0 & 22.2 & 70.5 \\
\hline \multirow{3}{*}{ Com atraso } & Tratamento & 10.7 & 26.9 & 152.0 & 8.0 & 19.5 & 144.7 \\
\hline & Controle 1 & 12.8 & 18.8 & 47.0 & 8.7 & 12.7 & 46.2 \\
\hline & Controle 2 & 12.6 & 18.9 & 50.0 & 8.2 & 12.2 & 49.2 \\
\hline \multirow{3}{*}{ Sem atraso } & Tratamento & 20.6 & 45.6 & 121.0 & 18.0 & 39.5 & 120.0 \\
\hline & Controle 1 & 18.7 & 31.3 & 67.3 & 16.0 & 25.3 & 58.0 \\
\hline & Controle 2 & 18.4 & 32.8 & 78.2 & 15.6 & 26.9 & 72.9 \\
\hline \multirow{3}{*}{ Trabalha fora } & Tratamento & 8.5 & 23.8 & 180.9 & 6.1 & 16.4 & 171.4 \\
\hline & Controle 1 & 8.0 & 14.3 & 79.4 & 5.8 & 9.0 & 55.7 \\
\hline & Controle 2 & 7.0 & 14.9 & 113.1 & 5.2 & 9.2 & 75.2 \\
\hline \multirow{3}{*}{$\begin{array}{l}\text { Não trabalha } \\
\text { fora }\end{array}$} & Tratamento & 20.4 & 46.4 & 127.5 & 16.6 & 37.7 & 127.4 \\
\hline & Controle 1 & 18.9 & 32.2 & 70.6 & 14.8 & 24.7 & 66.6 \\
\hline & Controle 2 & 18.8 & 33.9 & 80.2 & 14.5 & 26.0 & 79.0 \\
\hline \multirow{3}{*}{ Cursou El } & Tratamento & 20.8 & 44.8 & 115.6 & 16.6 & 35.6 & 114.4 \\
\hline & Controle 1 & 19.1 & 31.1 & 63.1 & 14.8 & 23.0 & 55.4 \\
\hline & Controle 2 & 18.5 & 32.5 & 75.9 & 14.2 & 24.1 & 70.0 \\
\hline \multirow{3}{*}{ Não cursou El } & Tratamento & 13.4 & 37.4 & 178.3 & 10.5 & 26.7 & 155.6 \\
\hline & Controle 1 & 12.8 & 23.0 & 79.7 & 9.5 & 15.7 & 65.6 \\
\hline & Controle 2 & 13.2 & 24.5 & 86.5 & 9.3 & 16.5 & 77.2 \\
\hline
\end{tabular}

Fonte: Elaboração das autoras com dados da Prova Brasil/Inep. 
Seja $y$ uma variável binária que assume valor 1 quando o aluno tem nota de LP igual ou maior a 200 e assume valor 0 , caso contrário. Para verificar se o Paic aumentou a equidade assim como entendida por Ribeiro (2012), estimaremos a seguinte equação:

$$
\operatorname{Pr}\left(y_{i s t}=1 \mid \gamma_{e}, \lambda_{t}, D_{e t}, X_{i t}\right)=\gamma_{e}+\lambda_{t}+\beta D_{e t}+\delta X_{i t}
$$

onde $i$ indexa o aluno, $\gamma_{e}$ é um efeito fixo do estado $e, \lambda_{t}$ é uma tendência temporal comum aos estados, $D_{e t}$ é uma variável dummy que indica o grupo tratado (estado do Ceará) em 2011, $X_{i t}$ é um vetor que pode conter características dos alunos (classe econômica, sexo, raça, atraso escolar, se trabalha fora de casa e se cursou educação infantil) e das escolas (características dos alunos agregadas por escola). Usamos o estimador de efeito fixo e o software Stata 13. Incluímos características dos alunos para aumentar a precisão do estimador (ANGRIST; PISCHKE, 2008) e, para corrigir a correlação serial associada, estimamos a matriz de covariância com cluster no estado. O NSE dos alunos foi estimado com base no Critério Brasil, vigente de 2008 a 2011. Para mais detalhes, consulte Padilha et al. (2013).

Estimamos também dois modelos para o painel de escolas que tinham, pelo menos, 10 alunos de interesse em 2007 e 2011 - o primeiro para verificar o efeito de tratamento sobre os tratados do Paic:

$$
Y_{j e t}=\rho_{j}+\gamma_{e}+\lambda_{t}+\beta D_{j t}+\delta X_{j t}+\varepsilon_{j e t}
$$

O segundo modelo de painel tem o objetivo de verificar se o programa tem efeitos heterogêneos a depender da classe econômica dos alunos no período pré-tratamento e se baseia em Galiani et al. (2008) e Leme et al. (2012):

$$
Y_{j e t}=\rho_{j}+\gamma_{e}+\lambda_{t}+\beta_{1} D_{j t} P_{j 07}+\beta_{2} D_{j t}+\delta X_{j t}+\varepsilon_{j e t}
$$

A escola está indexada por $j, Y_{j e t}$ é a proporção dos alunos de interesse que atingiram o piso de desempenho e $X_{j t}$ contém características desses alunos agregadas nas escolas. 
O estimador usado foi o de primeiras diferenças. Os demais termos são análogos ao da equação anterior. $P_{j 07}$ é uma variável binária que indica se a maioria dos alunos é pobre em 2007, de acordo com duas definições: (i) é considerada pobre a criança com classe econômica D ou E; (ii) é considerada pobre a criança com classe econômica C2, D ou E.

A Tabela 4 mostra os efeitos de tratamento do Paic (equações 1 e 2). Tanto no nível do aluno quanto no da escola, em relação a ambos os grupos de controle, o Paic aumentou a probabilidade de uma criança atingir o nível adequado de proficiência em LP. Os coeficientes variam de 9,6 a 13 pontos percentuais (p.p.). Considerando-se que o percentual de alunos com desempenho acima ou igual ao piso aumentou 25 p.p. entre 2007 e 2011 para a coorte (Tabela 2), pode-se dizer que o Paic foi responsável por, pelo menos, 39\% dessa elevação.

TABELA 4 - Estimativas (e erros padrão) dos parâmetros do modelo utilizado para avaliar o efeito do Paic

\begin{tabular}{|c|c|c|c|c|c|}
\hline NÍVEL & PARÂMETROS & $\begin{array}{c}\text { GRUPO } \\
\text { CONTROLE }\end{array}$ & ESTIMATIVAS & E.P. & $\mathrm{N}$ \\
\hline \multirow{6}{*}{ Alunos } & Efeitos fixos & \multirow{3}{*}{1} & 0.1306 & 0.0071 & 291.340 \\
\hline & Efeitos fixos e características dos alunos & & 0.1172 & 0.0054 & 248.939 \\
\hline & Efeitos fixos e características dos alunos e das escolas & & 0.1095 & 0.0043 & 248.939 \\
\hline & Efeitos fixos & \multirow{3}{*}{2} & 0.1140 & 0.0137 & 157.459 \\
\hline & Efeitos fixos e características dos alunos & & 0.1013 & 0.0084 & 135.570 \\
\hline & Efeitos fixos e características dos alunos e das escolas & & 0.0957 & 0.0038 & 135.570 \\
\hline \multirow{4}{*}{ Escolas } & Efeitos fixos & \multirow{2}{*}{1} & 0.1330 & 0.0084 & 4.715 \\
\hline & Efeitos fixos e características das escolas & & 0.1196 & 0.0063 & 4.715 \\
\hline & Efeitos fixos & \multirow{2}{*}{2} & 0.1190 & 0.0134 & 2.509 \\
\hline & Efeitos fixos e características das escolas & & 0.1066 & 0.0079 & 2.509 \\
\hline
\end{tabular}

Fonte: Elaboração das autoras com dados da Prova Brasil/Inep.

Nota: Variância com cluster no estado; p-valores são todos inferiores a 0.01

Os efeitos heterogêneos do Paic estão na Tabela 5 (equação 3) e vemos que o programa teve maior efeito na proporção de alunos que atingiram a nota mínima de escolas pobres, seja qual for a medida. O impacto para escolas não 
pobres varia de 9 a 11 p.p. e a diferença entre essas e as escolas pobres varia, grosso modo, de 2 a 6 p.p. Esse resultado é importante, visto que o percentual de alunos mais pobres que têm notas adequadas é menor.

TABELA 5 - Estimativas (e erros padrão) dos parâmetros do modelo utilizado para avaliar se o efeito do Paic é homogêneo ou se depende da classe econômica dos alunos

\begin{tabular}{|c|c|c|c|c|c|c|}
\hline \multirow{2}{*}{ PARÂMETROS } & \multirow{2}{*}{$\begin{array}{c}\text { GRUPO } \\
\text { CONTROLE }\end{array}$} & \multicolumn{2}{|c|}{ POBRES } & \multicolumn{2}{|c|}{ NÃO POBRES } & \multirow{2}{*}{$\mathrm{N}$} \\
\hline & & ESTIMATIVAS & E.P. & ESTIMATIVAS & E.P. & \\
\hline \multicolumn{7}{|c|}{ Definição de pobreza (1) } \\
\hline Efeitos fixos & \multirow{2}{*}{1} & 0.1782 & 0.0084 & 0.1121 & 0.0084 & 4.715 \\
\hline Efeitos fixos e características das escolas & & 0.1494 & 0.0078 & 0.1091 & 0.0061 & 4.715 \\
\hline Efeitos fixos & \multirow{2}{*}{2} & 0.1642 & 0.0134 & 0.0981 & 0.0134 & 2.509 \\
\hline Efeitos fixos e características das escolas & & 0.1402 & 0.0098 & 0.0969 & 0.0075 & 2.509 \\
\hline \multicolumn{7}{|c|}{ Definição de pobreza (2) } \\
\hline Efeitos fixos & \multirow{2}{*}{1} & 0.1424 & 0.0084 & 0.1073 & 0.1424 & 4.715 \\
\hline Efeitos fixos e características das escolas & & 0.1263 & 0.0069 & 0.1069 & 0.0057 & 4.715 \\
\hline Efeitos fixos & \multirow{2}{*}{2} & 0.1284 & 0.0134 & 0.0933 & 0.0134 & 2.509 \\
\hline Efeitos fixos e características das escolas & & 0.1144 & 0.0092 & 0.0938 & 0.0058 & 2.509 \\
\hline
\end{tabular}

Fonte: Elaboração das autoras com dados da Prova Brasil/Inep.

Nota: Variância com cluster no estado; p-valores são todos inferiores a 0.01

Com base nos resultados apresentados nesta seção, pode-se dizer que houve melhoria de equidade no Ceará para as crianças da coorte analisada, pois o Paic ajudou a aumentar a proporção de alunos da coorte que atinge o desempenho adequado em LP, especialmente em escolas com maioria de alunos pobres. Cabe lembrar que olhamos para uma parcela de alunos tratados pelo Paic em 2008, pois só fizeram a Prova Brasil em 2011 crianças que não reprovaram, que não migraram de estado e que não saíram da rede pública.

\section{CONSIDERAÇÕES FINAIS}

Este artigo buscou verificar se o Paic contribuiu para a melhoria da equidade em termos de proficiência em LP nos anos iniciais do EF na rede pública cearense. O conceito de 
equidade usado foi o de Ribeiro (2012), no qual equidade é entendida como a situação em que todos os alunos, independentemente de sua situação de origem, atingem níveis adequados de resultados.

Ao descrevermos e analisarmos a política, evidenciou-se foco na vigilância sobre processos de implementação e resultados educacionais no contexto cearense, elementos que a literatura afirma favorecer a promoção da equidade: a existência de um sistema de monitoramento da aprendizagem de cada aluno, ações de planejamento e acompanhamento nas escolas e outros níveis administrativos, indução de reorganização de processos nas redes municipais, formação de professores, uso de material estruturado, indução de políticas para a educação infantil, interpretação pedagógica dos resultados das avaliações externas na rede para correção de rumos e apoio adicional aos municípios com resultados mais baixos. Essas ações se aproximam do que foi nominado por Crahay (2000) de "sistema de pilotagem", necessário, segundo o autor, para a consecução da equidade.

O Paic ampliou a equidade no Ceará para as crianças da coorte analisada, pois ajudou a aumentar a proporção de alunos que atinge o desempenho mínimo em LP, principalmente em escolas com maioria de alunos pobres. Estimamos que o programa tem efeito mínimo de 9 p.p. para escolas não pobres e impacto adicional para escolas pobres que varia entre 2 e 6 p.p. Dois pontos importantes não puderam ser abordados neste artigo. $O$ primeiro deles diz respeito às diferenças de proficiência entre alunos das zonas rural e urbana. Atualmente, $27 \%$ da população cearense residem no campo (INSTITUTO BRASILEIRO DE GEOGRAFIA E ESTATÍSTICA IBGE, 2015). Historicamente, os estados do Nordeste apresentam alto percentual de população rural, cujos resultados educacionais são mais baixos. O segundo ponto é verificar o efeito do Paic sobre o fluxo escolar, pois se houve aumento de aprendizagem das crianças, conforme apontam nossos resultados e também os de outros autores, a probabilidade de reprovação pode ter sido reduzida. 


\section{REFERÊNCIAS}

ANGRIST, Joshua D.; PISCHKE, Jörn-Steffen. Mostly harmless econometrics: an empiricist's companion. Princeton: Princeton University, 2008.

BERNSTEIN, Basil. Classes e pedagogia: visível e invisível. Cadernos de Pesquisa, São Paulo, n. 49, p. 26-42, maio 1984.

BLUNDELL, Richard; DIAS, Monica Costa. Alternative approaches to evaluation in empirical microeconomics. The Journal of Human Resources, Wisconsin, v. 44, n. 3, p. 565-640, Summer 2009.

BRASIL. Ministério da Educação. Instituto Nacional de Estudos e Pesquisas Educacionais Anísio Teixeira. Saeb 2005: primeiros resultados: médias de desempenho do Saeb 2005 em perspectiva comparada. Fev. 2007. Disponível em: <http://download.inep.gov.br/educacao_basica/prova_ brasil_saeb/menu_do_professor/resultados/Saeb_resultados95_05_UF.pdf > . Acesso em: 16 jan. 2015.

BRASIL. Ministério da Educação. Instituto Nacional de Estudos e Pesquisas Educacionais Anísio Teixeira. Brasília, DF: Inep/MEC, 2015. Disponível em: $<$ http://portal.inep.gov.br/>. Acesso em: 16 jan. 2015.

CAMPOS, Maria Malta. Educação infantil: o debate e a pesquisa. Cadernos de Pesquisa, São Paulo, n. 101, p. 113-127, jul. 1997.

CEARÁ. Secretaria de Estado da Educação. Regime de colaboração para a garantia do direito à aprendizagem: o Programa Alfabetização na Idade Certa (Paic) no Ceará. Fortaleza: SEE, 2012. Disponível em: <http://www.paic.seduc. ce.gov.br/images/biblioteca/livro_regime_de_colaboracao.pdf. Acesso em: 2 mar. 2015.

CEARÁ. Secretaria de Estado da Educação. Ações desenvolvidas (2007/2010). Fortaleza: SEE, 2015a. Disponível em: <http://www.paic.seduc.ce.gov.br/ images/PASTA_TATIANA/acoes_paic_2007_a_2011.pdf>. Acesso em: 2 mar. 2015.

CEARÁ. Secretaria de Estado da Educação. Paic - Programa de Alfabetização na Idade Certa. Fortaleza: SEE, 2015b. Disponível em: <http://www.paic. seduc.ce.gov.br/>. Acesso em: 2 mar. 2015.

COSTA, Leandro; CARNOY, Martin. The effectiveness of an early grades literacy intervention on the cognitive achievement of Brazilian students. In: IZA/WORLD BANK CONFERENCE ON EMPLOYMENT AND DEVELOPMENT, 9., 2014, Lima. Disponível em: <http://www.iza.org/conference_files/ worldb2014/costa_110038.pdf>. Acesso em: 16 jan. 2015.

CRAHAY, Marcel. L'école peut-elle-être juste et efficace? De l'égalité des chances à l'égalité des acquis. Belgique: De Boeck, 2000.

DUBET, François. Les dilemmes de la justice. In: DEROUET, Jean-Louis ; DEROUET-BESSON, Jean-Louis. Repenser la justice dans le domaine del'éducation et de la formation. Lyon: PeterLang, 2009. p. 29-46.

GALIANI et al. School decentralization: helping the good get better, but leaving the poor behind. 2008. Disponível em: <http://ssrn.com/abstract=2450613>. Acesso em: 7 dez. 2015. 
GUSMÃO, Joana Buarque de; RIBEIRO, Vanda Mendes. Colaboração entre estado e municípios para a alfabetização de crianças na idade certa no Ceará. Cadernos Cenpec, São Paulo, v. 1, n. 1, p. 9-34, dez. 2011.

HOLLIDAY, Oscar Jara. Guia para sistematizar experiências. In: CENTRO DE INTERVENÇÃO PARA O DESENVOLVIMENTO AMÍLCAR

CABRAL. Sistematização de experiências: aprender a dialogar com os processos. Lisboa: Cidac, 2012. p. 5-35.

INSTITUTO BRASILEIRO DE GEOGRAFIA E ESTATÍSTICA. Sistema IBGE de Recuperação Automática: Sidra. 2015. Disponível em: <http://www.sidra. ibge.gov.br/>. Acesso em: 16 jan. 2015.

LAVOR, Daniel Campos; ARRAES, Ronaldo de Albuquerque de. Qualidade da educação básica e uma avaliação de política educacional para o Ceará. In: ENCONTRO ECONOMIA DO CEARÁ EM DEBATE, 10., 2014, Fortaleza. Anais... Fortaleza, Instituto de Pesquisa e Estratégia Econômica do Ceará IPECE, 2014.

LEME, Maria Carolina; LOUZANO, Paula; PONCZEK, Vladimir; SOUZA, André Portela. The impact of structured teaching methods on the quality of education in Brazil. Economics of Education Review, Amsterdam, v. 31, n. 5, p. 850-860, Oct. 2012.

PADILHA, Frederica V. Q.; BATISTA, Antônio Augusto Gomes. Evolução dos indicadores educacionais no Ceará: os anos finais do ensino fundamental. São Paulo: Cenpec, 2013. (Informe de Pesquisa, n. 4).

PADILHA, Frederica V. Q. et al. Os municípios e a qualidade das escolas na segunda etapa do ensino fundamental. São Paulo: Cenpec, 2011. (Informe de Pesquisa, n. 1).

PADILHA, Frederica V. Q. et al. Os municípios e a qualidade das escolas na segunda etapa do ensino fundamental: as regularidades e as exceções do desempenho no Ideb. Estudos em Avaliação Educacional, São Paulo, v. 23, n. 51, p. 58-81, jan./abr. 2012.

PADILHA, Frederica V. Q. et al. Qualidade e equidade no ensino fundamental público do Ceará. Cadernos Cenpec, São Paulo, v. 3, n. 1, p. 81-110, 2013.

RAWLS, John. Justiça como equidade: uma reformulação. São Paulo: Martins Fontes, 2003.

RIBEIRO, Vanda Mendes. Justiça na escola e regulação institucional de redes de ensino do estado de São Paulo. 2012. Tese (Doutorado) - Faculdade de Educação da Universidade de São Paulo, São Paulo, 2012.

RIBEIRO, Vanda Mendes. Que princípio de justiça para a educação básica? Cadernos de Pesquisa, São Paulo, v. 44, n. 154, p. 1094-1109, out./dez. 2014.

TODOS PELA EDUCAÇÃO. Nota técnica preliminar. Metodologia para a obtenção das metas finais e parciais. Todos pela Educação, 2007. Mimeografado. 
PAULA KASMIRSKI

Doutoranda pela Escola de Economia de São Paulo (EESP)/ Fundação Getúlio Vargas (FGV). Pesquisadora do Centro de Estudos e Pesquisas em Educação, Cultura e Ação Comunitária (Cenpec), São Paulo, São Paulo, Brasil prkasmirski@gmail.com

JOANA GUSMAO

Pesquisadora do Centro de Estudos e Pesquisas em Educação, Cultura e Ação Comunitária (Cenpec), São Paulo, São Paulo, Brasil

joana.buarque@cenpec.org.br

VANDA RIBEIRO

Professora dos Programas de Pós-graduação em Educação e Educação Profissional da Universidade Cidade de São Paulo (Unicid). Pesquisadora do Centro de Estudos e Pesquisas em Educação, Cultura e Ação Comunitária (Cenpec), São Paulo, São Paulo, Brasil.

vandaribeiro2@gmail.com 
